\title{
Keynote: Graph-based Approaches for Spoken Language Understanding
}

\author{
Dilek Hakkani-Tur \\ Microsoft Research, U.S.A. \\ dilek@ieee.org
}

Following an upsurge in mobile device usage and improvements in speech recognition performance, multiple virtual personal assistant systems have emerged, and have been widely adopted by users. While these assistants proved to be beneficial, their usage has been limited to certain scenarios and domains, with underlying language understanding models that have been finely tuned by their builders.

Simultaneously, there have been several recent advancements in semantic web knowledge graphs especially used for basic question answering, efforts for integrating statistical information on these graphs, graph-based generic semantic representations and parsers, all providing opportunities for open domain spoken language understanding.

In this talk, I plan to summarize recent work in these areas, focusing on their connection, as a promise for wide coverage spoken language understanding in conversational systems, while at the same time investigating what is still lacking for natural human-machine interactions and related challenges. 